\title{
Brain-Computer Interface in Stroke Rehabilitation
}

\author{
Kai Keng Ang* and Cuntai Guan \\ Institute for Infocomm and Research, Agency of Science, Technology and Research (A*STAR), Singapore \\ kkang@i2r.a-star.edu.sg, ctguan@i2r.a-star.edu.sg
}

\begin{abstract}
Recent advances in computer science enabled people with severe motor disabilities to use brain-computer interfaces (BCI) for communication, control, and even to restore their motor disabilities. This paper reviews the most recent works of BCI in stroke rehabilitation with a focus on methodology that reported on data collected from stroke patients and clinical studies that reported on the motor improvements of stroke patients. Both types of studies are important as the former advances the technology of BCI for stroke, and the latter demonstrates the clinical efficacy of BCI in stroke. Finally some challenges are discussed.
\end{abstract}

Category: Human computing

Keywords: Stroke; Rehabilitation; Brain-Computer Interface; Motor Imagery, Electroencephalography

\section{INTRODUCTION}

Stroke occurs when blood supply to the brain is blocked or when blood vessels in the brain burst. About 15 million people worldwide suffer from stroke every year [1]. One in 20 adults (aged $>14$ years) in the developed countries is affected by stroke [2]. Stroke is a major cause of disability and it affects the quality of life of the survivors in their daily functioning in the workplace, home, and community. Recovery of the motor function after stroke is crucial in order to perform activities of daily living, but this recovery is often variable and incomplete [3]. The main mechanism underlying motor recovery involves enhanced activity of the primary motor cortex induced by Active Motor Training (AMT) and pharmacological interventions [4]. Clinical studies of AMT such as constraint-induced movement therapy (CIMT) improve outcome after chronic stroke, but poor motor performance excludes most of the otherwise eligible acute stroke patients from CIMT [5]. Hence, the clinical use of AMT is limited by a dependence on residual motor performance.

Since physical movements by stroke patients are often not possible, alternate strategies are needed. Motor imagery (MI), the mental rehearsal of physical movement tasks, represents a new approach to access the motor system and rehabilitation at all stages of stroke recovery [6]. Unlike AMT, MI is not dependent on residual motor performance, and direct cellular recording of primate has shown that the primary motor cortex (M1) is involved during MI [7]. A functional imaging study in subcortical stroke had shown that the motor system is activated during MI despite the lesion [8]. Since stroke does not impair the capacity to perform MI $[9,10]$, MI provides a substitute for AMT as a means to activate the motor network in stroke [6]. However, while AMT can be observed, MI is concealed within the patient. Thus it is difficult to assess the performance of MI non-invasively without involving functional magnetic resonance imaging (fMRI), positron emission tomography (PET), near infrared spec-

This is an Open Access article distributed under the terms of the Creative Commons Attribution Non-Commercial License (http:/creativecommons.org/licenses/ by-nc/3.0/) which permits unrestricted non-commercial use, distribution, and reproduction in any medium, provided the original work is properly cited. 
troscopy (NIRS), scalp-recorded magnetoencephalography (MEG) or electroencephalography (EEG) [6].

A brain-computer interface (BCI), or sometimes called brain-machine interface, is a device that responds to neural processes from the brain to provide a direct communication pathway between the brain and the external device [11] without the use of the normal neuromuscular pathways. BCI technology can substantially improve the lives of people with devastating neurological disorders, such as advanced amyotrophic lateral sclerosis, restore more effective motor control to people after stroke or other traumatic brain disorders, by guiding activity-dependent brain plasticity by using brain signals to indicate the state of brain activity [12]. This technology can also be used to supplement impaired muscle control, or to increase the efficacy of a rehabilitation protocol to improve muscle control of the patient.

This paper reviews the current works of BCI in stroke. We first briefly discuss works that provided reviews of $\mathrm{BCI}$ studies in the literature, followed by our review of the relevant works in chronological order with emphasis on methodology studies that reported on data collected from stroke patients and clinical studies that focused on rehabilitation whereby the efficacy in motor recovery or neuroimaging evidence were reported. We also present a summary table to comprehensively list and compare the relevant works. Finally we address some challenges for the future prospect of $\mathrm{BCI}$ in stroke.

\section{BCI IN STROKE}

A BCI is a computer-based system that translates brain signals into commands for an output device to perform a desired action. A stricter definition of BCI is limited to systems that only use signals from the central nervous system (CNS), and systems that are voice or muscle-activated are not considered BCI [13]. As such, the main interest in BCI technology is to help people who suffered from neurological disorders such as amyotrophic lateral sclerosis, stroke or other traumatic brain disorders [12]. There are two ways BCI technology can help stroke survivors. The first way, which has been the focus of considerable research in the past, is to substitute for the loss of neuromuscular functions by using stroke survivors' brain signals to interact with the environment instead of using their impaired muscles. For example, a BCI can be used by a stroke survivor to control a computer cursor or a limb orthosis, for word processing and accessing the internet, and for other functions such as environmental control or entertainment. The second way, which has only recently been studied, is to use BCI for stroke rehabilitation in order to restore the impaired motor function. Since stroke affects parts of the brain but not the impaired limbs, BCI could be used to induce and guide activity- dependent brain plasticity of the stroke survivors by paying close attention to a motor task that required the activation or deactivation of specific brain signals.

The theory behind AMT in stroke rehabilitation is called CIMT [5]. This theory is based on the idea of "learned non-use", which develops during the early stages of the stroke survivor to compensate for difficulty in using the impaired limb from increased reliance on the intact limb. Rehabilitation therapy thus involves immobilizing the intact limb while the stroke survivor is trained to use the impaired limb. The theory behind BCI stroke rehabilitation is similar to CIMT, but the difference lies in focusing on thoughts of moving the impaired limb instead of physically moving the impaired limb. This is called MI, which is the mental rehearsal of physical movement tasks. For many stroke patients, moving the impaired limb is very difficult and sometimes impossible even after early participation in an active rehabilitation program. Thus MI can be used to perform repetitions of movement at the cerebral level without any physical demands [14]. In other words, the intervention from AMT is primarily targeted at the stroke impaired limb, whereas the intervention from MI is primarily targeted at the stroke impaired brain. Since the performance of MI is not dependent on any residual motor performance of the impaired limb, this represents an alternate approach [6]. There is scientific evidence that the primary motor cortex is involved during MI as well as motor execution (ME), thus the capacity to perform MI is not impaired by stroke and may be substituted for ME as a means to activate the motor network in stroke. However, ME can be checked by observation, whereas MI is concealed within the patient. Nevertheless, BCI can be used to objectively assess the performance of MI. Neurophysiologists have shown that the performance of MI and ME result in compression of the $\mu$ - or $\beta$-rhythms in EEG brain signals. This phenomenon is known as event-related synchronization (ERS) or event-related desynchronization (ERD), and various BCI-based have been developed to detect this from EEG during MI. The challenge arises in the detection of MI due to the low signal-to-noise ratio, and recent advances in signal processing and machine learning methods have enabled the clinical deployment of BCI for stroke rehabilitation.

\section{REVIEWS ON BCI}

There are currently several recent reviews of BCI in the literature. The recent review by Nicolas-Alonso and Gomez-Gil [15] comprehensively covered the different BCI modalities, such as EEG, MEG, fMRI, NIRS, electrocorticography (ECoG), and intracortical neuron recording. This review also broadly discussed how certain neurophysiological signals can be used to determine 
the user intentions, such as visual evoked potentials (VEPs), slow cortical potentials (SCPs), P300 evoked potentials, and sensorimotor rhythms. In addition, the review also presented some feature extraction and classification techniques used in $\mathrm{BCI}$ applications that translate information in the control signals into commands to operate a computer or other device, and provided an overview of various BCI applications that control a range of devices.

Another review by Shih et al. [13] discussed the application of BCI in the field of Medicine, and stated three critical areas of development in the future of BCIs: comfortable, convenient, and stable signal-acquisition hardware; $\mathrm{BCI}$ validation and dissemination; and proven $\mathrm{BCI}$ reliability and value for various user populations. Silvoni et al. [16] presented a review on the history of BCI research in stroke up to 2011, and classified BCI-based rehabilitation into three approaches: substitutive strategy, classical conditioning and operant conditioning. In addition, they also presented their pilot study of a new neuroforcefeedback strategy on healthy subjects.

The review by Silvoni et al. [16] is one of the most comprehensive review on the progress of BCI in stroke up to 2011, and also covered works that reported on healthy subjects, patients with spinal cord injury (SCI) and amyotrophic lateral sclerosis (ALS). Since 2011, there is a significant increase in the research of BCI in stroke. Thus we review works up to 2013, focusing only on works that reported data collected from stroke.

\section{RECENT WORKS OF BCI ON STROKE PATIENTS}

Table 1 presents a review on studies of BCI on stroke in a chronological order. A search revealed numerous papers of BCI for stroke, but only those papers that reported work on data collected from stroke patients are included in this review. These studies are classified into the type BCI modalities (EEG, MEG, NIRS as well as any other signals such as eye trackers or stimulation such as transcranial direct current stimulation [tDCS]) and type of BCI feedback (visual, robot, orthosis, functional electrical stimulation [FES]). In addition, the number of stroke patients included in these studies, the type of task (MI or ME), the targeted stroke-affected disability (hand, upper limb, lower limb, ankle), and whether physical therapy is included are summarized. Most importantly, the types of motor assessment used to evaluate the effectiveness of the BCI intervention are summarized. In addition, whether randomized control trials (RCT) were conducted, the most rigorous way of evaluating an intervention and its effectiveness [17] is indicated. Furthermore, any other evidence in neuroimaging that showed the effectiveness of the BCI intervention is also summarized. Finally, works that reported on data collected from the same clinical study are annotated.

\section{A. Methodology Studies}

The first attempt to use BCI in motor rehabilitation [16] was presented by Pfurtscheller et al. [18] on a patient who suffered from traumatic spinal cord injury, but not on one who suffered from stroke. Subsequently, the first study of BCI on stroke patients [16] was first reported by Mohapp et al. [19]. This study focused on extracting relevant features from EEG of stroke patients from performing MI and ME of hand. We classified such a study as methodology studies, which focused more on the methodology than the motor outcome. Methodology studies are important as they advance the technology of BCI in stroke. Similarly, Bai et al. [20] reported the performance of a sensorimotor $\beta$-rhythm-based BCI with visual feedback but without BCI training on a stroke patient. The other difference between this study and the first study was that visual feedback was employed, which is an important aspect of BCI.

Subsequently, there was an increase in the number of papers that reported methodology studies on stroke patients [21-31], and henceforth we will only briefly highlight some of these studies that we found exhibited distinctiveness from other studies. Besides targeting the hand and the upper limb functions, Niazi et al. [23] reported a study on 5 stroke patients targeting ME of the lower limb ankle function. Although this study used a BCI system that was muscle-activated and thus strictly not considered a BCI [13], this was the first study on stroke patients that targeted one of the other four MI actions other than left and right upper limb [32]. Tam et al. [24] and subsequently Arvaneh et al. [25, 26] presented methods that specifically reduced the number of EEG electrodes of MI BCI for stroke subjects. Using BCI with a large number of channels involves a longer preparation time that directly impacts the convenience of stroke patients using BCI for rehabilitation. These two works thus directly addressed this issue, which is essential in the use of BCI for stroke patients in the clinical setting.

\section{B. Clinical Studies}

The very first clinical study of using BCI on stroke patients was presented by Buch et al. [33]. In this study, 8 patients modulated their m-rhythm to control a screen cursor using a MEG-based BCI. Although 6 out of 8 were successful in controlling the screen cursor linked to an orthosis attached to the stroke-affected hand, no significant improvements in motor outcome were reported using the Medical Research Council (MRC) scale. We classified such a study that reported clinical measures in motor outcome as clinical studies. Clinical studies are very important as they demonstrate the clinical efficacy of BCI in stroke.

Next, Ang et al. [34] reported preliminary outcome of an RCT to investigate the outcome of MI BCI robotic 


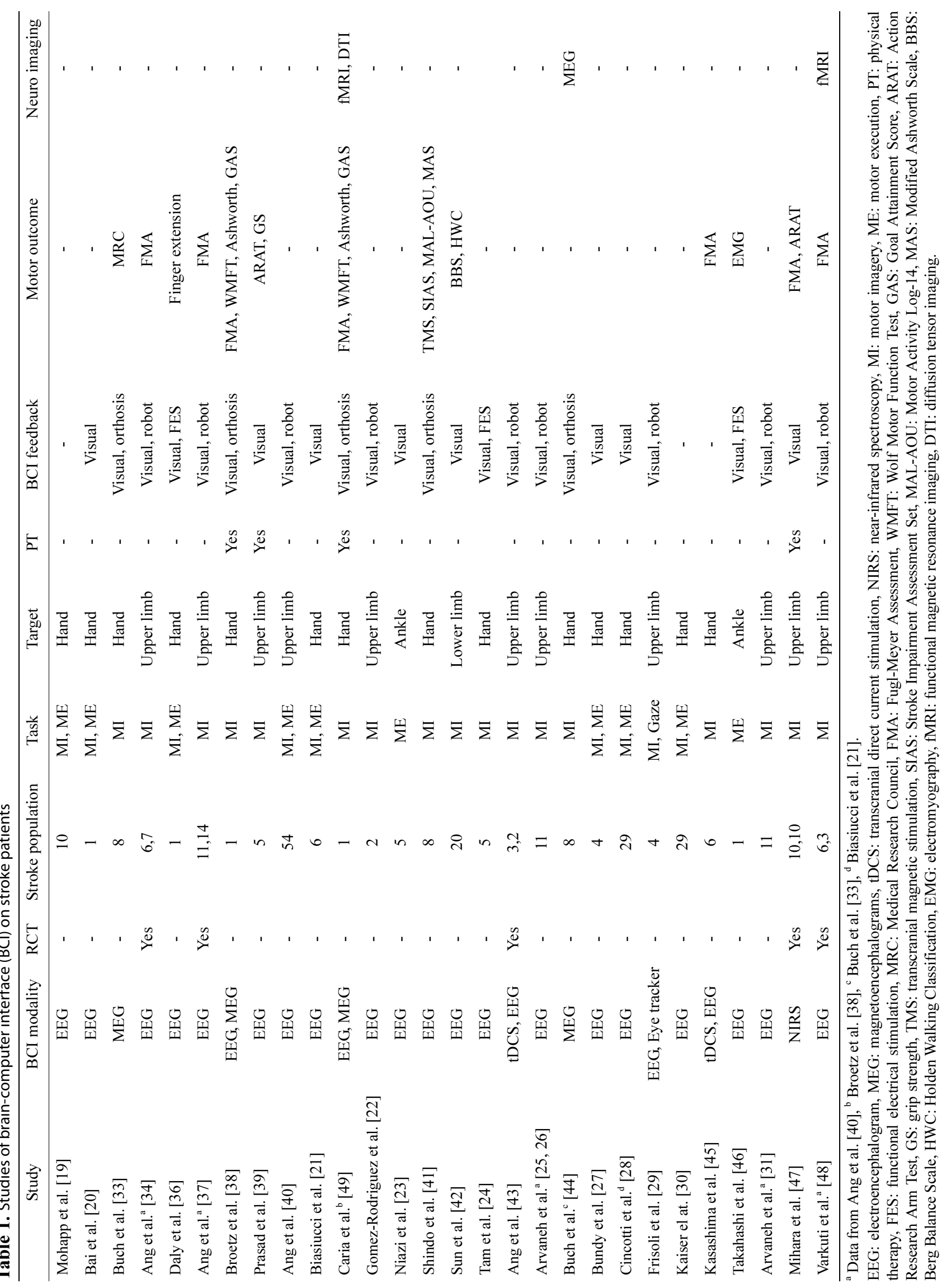


intervention compared to robotic intervention for upper limb rehabilitation on 6 and 7 stroke patients, respectively. The motor outcome was clinically measured using the Fugl-Meyer Assessment (FMA) [35], and the study showed statistically significant motor outcome improvements in both groups after 12 sessions of 1-hour rehabilitation for 4 weeks, but no significant difference between groups were found. This was the first clinical study to show significant improvements in motor outcome from BCI intervention. In another study, Daly et al. [36] reported the clinical outcome of BCI for both $\mathrm{MI}$ and $\mathrm{ME}$ with functional electrical stimulation (FES) feedback intervention on 1 stroke patient, and the patient demonstrated recovery measured by increased finger extension after 9 sessions of intervention, 3 times per week for 3 weeks. Next, Ang et al. [37] updated the outcome of the RCT preliminarily reported in [34] on 11 and 14 stroke patients, respectively. Broetz et al. [38] reported a case study of using EEG and MEG-based BCI with visual and orthosis feedbacks intervention coupled with daily lifeoriented physiotherapy on 1 stroke patient for a year, and motor improvements measured using a battery of assessments such as FMA, Wolf Motor Function Test (WMFT), Ashworth, 10-m Walk and Goal Attainment Score (GAS), etc. showed positive improvements. This was one of the first few studies that coupled BCI-based intervention with physical therapy. In another study, Prasad et al. [39] also reported MI BCI intervention coupled with physical therapy on 5 stroke patients, and outcome measures using Action Research Arm Test (ARAT) and grip strength (GS) showed positive improvement in at least one of the outcome measures in all the subjects.

Furthermore, Ang et al. [40] presented a study on the extent of detectable brain signals from a large population of 54 stroke patients in using EEG-based BCI to perform MI of upper limb. This study showed that a majority of but not all stroke patients could use EEG-based MI BCI, and hence suggested that a BCI screening is required to screen the stroke patient's capability of using BCI before enrolling them for BCI-based stroke rehabilitation intervention.

Subsequently, there was an increase in the number of papers that reported clinical studies on stroke patients [41-48]. Similar to the methodology studies, we will only highlight some of these studies that we found to exhibit distinctiveness from the other studies. Caria et al. [49] presented a case report of EEG and MEG-based BCI intervention coupled with physiotherapy on a stroke patient, and showed evidence of recovery as a result of brain plasticity using DTI and fMRI. This was the first case study that showed both efficacy in terms of clinical motor improvements as well as neuroimaging. In addition, Varkuti et al. [48] reported an fMRI RCT study on 6 stroke patients who underwent MI BCI with robotic feedback compared to 3 stroke patients who received robotic rehabilitation. The results showed functional connectivity changes that were numerically higher in the group that received BCI with robotic feedback intervention. These two studies both showed brain plasticity evidence as a result from BCI intervention in stroke.

Next, Ang et al. [43] presented the preliminary result of RCT study that coupled tDCS and MI EEG-based BCI with robotic feedback intervention versus the sham-tDCS group on 2 and 3 stroke patients, respectively. The results of the study showed higher BCI accuracies from subjects who received tDCS compared to sham-tDCS, though not statistically conclusive. In a separate study, Kasashima et al. [45] also presented the result of coupling tDCS with EEG-based BCI, and demonstrated that anodal tDCS resulted in an increase in ERD of the stroke-affected hemisphere in stroke patients. The results from these two studies seem to suggest tDCS effect in modulating MI in stroke patients.

Last but not least, Mihara et al. [47] recently presented the results of an RCT on 10 stroke patients who received NIRS-based BCI with visual feedback versus 10 stroke patients who comprised the sham group and received NIRS-based BCI with irrelevant feedback. The results showed that the patients who received BCI visual feedback showed significantly greater motor improvements measured using FMA compared to the sham group. This is an important study that showed clinical evidence that BCI feedback is effective and important in BCI stroke rehabilitation.

\section{DISCUSSION}

BCI has attracted increased interest, especially in stroke rehabilitation. This paper reviewed the most recent works of BCI in stroke that reported on data collected from patients, and grouped the studies into methodology studies and clinical studies. Both are equally important as the former advances the technological development of BCI in stroke, and the latter demonstrates the efficacy of BCI in stroke.

Although BCI technology has the prospect of improving the lives of people with neurological disorder, or restoring motor control to stroke patients [12], there were relatively more methodology studies on healthy subjects than on stroke patients. Nevertheless, it is promising to see works that research on methods to reduce the number of EEG channels for stroke patients' convenience while using BCI for stroke rehabilitation. In addition, many BCI methodology studies in stroke targeted the upper limb functions, and it is promising to see a few works that targeted the lower limb. Since tongue MI is one of the four MI actions detectable [32], there is also the prospect of using tongue MI for dysphagia rehabilitation.

Although there are an increasing number of clinical studies of BCI in stroke rehabilitation, there are some studies that reported both MI and ME. The dilemma is 
that $\mathrm{ME}$ is the current practice of physical therapy and robotic rehabilitation, but $\mathrm{BCI}$ that muscle-activated is not strictly considered as BCI [13]. Thus the motor improvements may be the result of ME and not MI. Furthermore, it is more practical for a stroke patient with residue movements to perform AMT instead of MI. Another dilemma exists on whether to couple BCI rehabilitation with physical therapy, since study had shown that the combination yielded motor improvement of chronic stroke patients [38], but it is difficult to separate the confounding motor improvements of physical therapy from the BCI rehabilitation. An RCT study could be performed to rigorously evaluate the $\mathrm{BCI}$ intervention versus another intervention such as physical therapy, but RCTs are more costly and time consuming to execute [17]. Hence researchers have to consider these quandaries before conducting a clinical study of BCI in stroke.

\section{CONCLUSIONS}

This paper reviewed the recent works of BCI in stroke patients specifically on methodology and clinical studies. The former focused on methods developed for and on data from stroke patients, and the latter focused on clinical studies to evaluate the efficacy of BCI in stroke. From the amount of recent works reviewed, the interest in BCI research for stroke is definitely increasing. Several challenges in the $\mathrm{BCI}$ for stroke rehabilitation research are discussed in this paper. Nevertheless, innovative developments are definitely forthcoming and increasing convincing clinical evidence of the efficacy of BCI in stroke will become available.

\section{ACKNOWLEDGMENTS}

Funded by the Science and Engineering Research Council of Agency for Science, Technology and Research (A*STAR), Singapore.

\section{REFERENCES}

1. R. Bene, N. Beck, B. Vajda, S. Popovic, K. Cosic, and V. Demarin, "Interface providers in stroke neurorehabilitation," Periodicum Biologorum, vol. 114, no. 3, pp. 403-407, 2012.

2. V. L. Feigin, C. M. M. Lawes, D. A. Bennett, S. L. BarkerCollo, and V. Parag, "Worldwide stroke incidence and early case fatality reported in 56 population-based studies: a systematic review," Lancet Neurology, vol. 8, no. 4, pp. 355369, 2009.

3. P. W. Duncan, L. B. Goldstein, D. Matchar, G. W. Divine, and J. Feussner, "Measurement of motor recovery after stroke: outcome assessment and sample size requirements," Stroke, vol. 23, no. 8, pp. 1084-1089, 1992.
4. C. Calautti and J. C. Baron, "Functional neuroimaging studies of motor recovery after stroke in adults: a review," Stroke, vol. 34, no. 6, pp. 1553-1566, 2003.

5. J. C. Grotta, E. A. Noser, T. Ro, C. Boake, H. Levin, J. Aronowski, and T. Schallert, "Constraint-induced movement therapy," Stroke, vol. 35, no. 11 (suppl 1), pp. 2699-2701, 2004.

6. N. Sharma, V. M. Pomeroy, and J. C. Baron, "Motor imagery: a backdoor to the motor system after stroke?," Stroke, vol. 37, no. 7, pp. 1941-1952, 2006.

7. A. P. Georgopoulos, J. T. Lurito, M. Petrides, A. B. Schwartz, and J. T. Massey, "Mental rotation of the neuronal population vector," Science, vol. 243, no. 4888, pp. 234-236, 1989.

8. N. Sharma, L. H. Simmons, P. S. Jones, D. J. Day, T. A. Carpenter, V. M. Pomeroy, E. A. Warburton, and J. C. Baron, "Motor imagery after subcortical stroke: a functional magnetic resonance imaging study," Stroke, vol. 40, no. 4, pp. 1315-1324, 2009.

9. S. H. Johnson, "Imagining the impossible: intact motor representations in hemiplegics," NeuroReport, vol. 11, no. 4, pp. 729-732, 2000.

10. S. H. Johnson, G. Sprehn, and A. J. Saykin, "Intact motor imagery in chronic upper limb hemiplegics: evidence for activity-independent action representations," Journal of Cognitive Neuroscience, vol. 14, no. 6, pp. 841-852, 2002.

11. J. R. Wolpaw, N. Birbaumer, D. J. McFarland, G. Pfurtscheller, and T. M. Vaughan, "Brain-computer interfaces for communication and control," Clinical Neurophysiology, vol. 113, no. 6, pp. 767-791, 2002.

12. J. J. Daly and J. R. Wolpaw, "Brain-computer interfaces in neurological rehabilitation," Lancet Neurology, vol. 7, no. 11, pp. 1032-1043, 2008.

13. J. J. Shih, D. J. Krusienski, and J. R. Wolpaw, "Brain-computer interfaces in medicine," Mayo Clinic Proceedings, vol. 87, no. 3, pp. 268-279, 2012.

14. P. L. Jackson, M. F. Lafleur, F. Malouin, C. Richards, and J. Doyon, "Potential role of mental practice using motor imagery in neurologic rehabilitation," Archives of Physical Medicine and Rehabilitation, vol. 82, no. 8, pp. 1133-1141, 2001.

15. L. F. Nicolas-Alonso and J. Gomez-Gil, "Brain computer interfaces: a review," Sensors, vol. 12, no. 2, pp. 1211-1279, 2012.

16. S. Silvoni, A. Ramos-Murguialday, M. Cavinato, C. Volpato, G. Cisotto, A. Turolla, F. Piccione, and N. Birbaumer, "Brain-computer interface in stroke: a review of progress," Clinical EEG and Neuroscience, vol. 42, no. 4, pp. 245-252, 2011.

17. S. Bonnie and R. Martin, "Understanding controlled trials: Why are randomised controlled trials important?," British Medical Journal, vol. 316, no. 7126, p. 201, 1998.

18. G. Pfurtscheller, G. R. Muller, J. Pfurtscheller, H. J. Gerner, and R. Rupp, “'Thought': control of functional electrical stimulation to restore hand grasp in a patient with tetraplegia," Neuroscience Letters, vol. 351, no. 1, pp. 33-36, 2003.

19. A. Mohapp, R. Scherer, C. Keinrath, P. Grieshofer, G. Pfurtscheller, and C. Neuper, "Single-trial EEG classification of executed and imagined hand movements in hemiparetic stroke patients," in Proceedings of the $3 \mathrm{rd}$ 
International Brain-Computer Interface Workshop and Training Course, Graz, Austria, 2006, pp. 80-81.

20. O. Bai, P. Lin, S. Vorbach, M. K. Floeter, N. Hattori, and M. Hallett, "A high performance sensorimotor beta rhythmbased brain-computer interface associated with human natural motor behavior," Journal of Neural Engineering, vol. 5, no. 1, pp. 24-35, 2008.

21. A. Biasiucci, R. Chavarriaga, B. Hamner, R. Leeb, F. Pichiorri, F. De Vico Fallani, D. Mattia, and J. R. del Millan, "Combining discriminant and topographic information in BCI: preliminary results on stroke patients," in Proceedings of the 5th International IEEE/EMBS Conference on Neural Engineering, Cancun, Mexico, 2011, pp. 290-293.

22. M. Gomez-Rodriguez, J. Peters, J. Hill, B. Schölkopf, A. Gharabaghi, and M. Grosse-Wentrup, "Closing the sensorimotor loop: haptic feedback facilitates decoding of motor imagery," Journal of Neural Engineering, vol. 8, no. 3, p. 036005, 2011.

23. I. K. Niazi, N. Jiang, O. Tiberghien, J. F. Nielsen, K. Dremstrup, and D. Farina, "Detection of movement intention from single-trial movement-related cortical potentials," Journal of Neural Engineering, vol. 8, no. 6, p. 066009, 2011.

24. W. K. Tam, K. Y. Tong, F. Meng, and S. K. Gao, "A minimal set of electrodes for motor imagery BCI to control an assistive device in chronic stroke subjects: a multi-session study," IEEE Transactions on Neural Systems and Rehabilitation Engineering, vol. 19, no. 6, pp. 617-627, 2011.

25. M. Arvaneh, C. Guan, K. K. Ang, and C. Quek, "Robust EEG channel selection across sessions in brain-computer interface involving stroke patients," in Proceedings of the IEEE International Joint Conference on Neural Networks, Brisbane, Australia, 2012, pp. 2319-2324.

26. M. Arvaneh, C. Guan, K. K. Ang, and C. Quek, "Omitting the intra-session calibration in EEG-based brain computer interface used for stroke rehabilitation," in Proceedings of the 34th Annual International Conference of the IEEE Engineering in Medicine and Biology Society, San Diego, CA, 2012, pp. 4124-4127.

27. D. T. Bundy, M. Wronkiewicz, M. Sharma, D. W. Moran, M. Corbetta, and E. C. Leuthardt, "Using ipsilateral motor signals in the unaffected cerebral hemisphere as a signal platform for brain-computer interfaces in hemiplegic stroke survivors," Journal of Neural Engineering, vol. 9, no. 3, p. 036011, 2012.

28. F. Cincotti, F. Pichiorri, P. Arico, F. Aloise, F. Leotta, F. D. Fallani, J. D. Millan, M. Molinari, and D. Mattia, "EEGbased brain-computer interface to support post-stroke motor rehabilitation of the upper limb," in Proceedings of the 34th Annual International Conference of the IEEE Engineering in Medicine and Biology Society, San Diego, CA, 2012, pp. 4112-4115.

29. A. Frisoli, C. Loconsole, D. Leonardis, F. Banno, M. Barsotti, C. Chisari, and M. Bergamasco, "A new gaze-BCIdriven control of an upper limb exoskeleton for rehabilitation in real-world tasks," IEEE Transactions on Systems Man and Cybernetics Part C, vol. 42, no. 6, pp. 1169-1179, 2012.

30. V. Kaiser, I. Daly, F. Pichiorri, D. Mattia, G. R. Muller-Putz, and C. Neuper, "Relationship between electrical brain responses to motor imagery and motor impairment in stroke," Stroke, vol. 43, no. 10, pp. 2735-2740, 2012.

31. M. Arvaneh, C. Guan, K. K. Ang, and C. Quek, "Optimizing spatial filters by minimizing within-class dissimilarities in electroencephalogram-based brain-computer interface," IEEE Transactions on Neural Networks and Learning Systems, vol. 24, no. 4, pp. 610-619, 2013.

32. M. Naeem, C. Brunner, R. Leeb, B. Graimann, and G. Pfurtscheller, "Seperability of four-class motor imagery data using independent components analysis," Journal of Neural Engineering, vol. 3, no. 3, pp. 208-216, 2006.

33. E. Buch, C. Weber, L. G. Cohen, C. Braun, M. A. Dimyan, T. Ard, J. Mellinger, A. Caria, S. Soekadar, A. Fourkas, and N. Birbaumer, "Think to move: a neuromagnetic brain-computer interface (BCI) system for chronic stroke," Stroke, vol. 39, no. 3, pp. 910-917, 2008.

34. K. K. Ang, C. Guan, K. S. G. Chua, B. T. Ang, C. W. K. Kuah, C. Wang, K. S. Phua, Z. Y. Chin, and H. Zhang, "A clinical study of motor imagery-based brain-computer interface for upper limb robotic rehabilitation," in Proceedings of the 31st Annual International Conference of the IEEE Engineering in Medicine and Biology Society, Minneapolis, MN, 2009, pp. 5981-5984.

35. A. R. Fugl-Meyer, L. Jaasko, I. Leyman, S. Olsson, and S. Steglind, "The post-stroke hemiplegic patient. 1. A method for evaluation of physical performance," Scandinavian Journal of Rehabilitation Medicine, vol. 7, no. 1, pp. 13-31, 1975.

36. J. J. Daly, R. Cheng, J. Rogers, K. Litinas, K. Hrovat, and M. Dohring, "Feasibility of a new application of noninvasive brain computer interface (BCI): a case study of training for recovery of volitional motor control after stroke," Journal of Neurologic Physical Therapy, vol. 33, no. 4, pp. 203211, 2009.

37. K. K. Ang, C. Guan, K. S. G. Chua, B. T. Ang, C. W. K. Kuah, C. Wang, K. S. Phua, Z. Y. Chin, and H. Zhang, "Clinical study of neurorehabilitation in stroke using EEGbased motor imagery brain-computer interface with robotic feedback," in Proceedings of the 32nd Annual International Conference of the IEEE Engineering in Medicine and Biology Society, Buenos Aires, Argentina, 2010, pp. 5549-5552.

38. D. Broetz, C. Braun, C. Weber, S. R. Soekadar, A. Caria, and N. Birbaumer, "Combination of brain-computer interface training and goal-directed physical therapy in chronic stroke: a case report," Neurorehabilitation and Neural Repair, vol. 24, no. 7, pp. 674-679, 2010.

39. G. Prasad, P. Herman, D. Coyle, S. McDonough, and J. Crosbie, "Applying a brain-computer interface to support motor imagery practice in people with stroke for upper limb recovery: a feasibility study," Journal of Neuroengineering and Rehabilitation, vol. 7, no. 1, p. 60, 2010.

40. K. K. Ang, C. Guan, K. S. G. Chua, B. T. Ang, C. W. K. Kuah, C. Wang, K. S. Phua, Z. Y. Chin, and H. Zhang, "A large clinical study on the ability of stroke patients to use EEG-based motor imagery brain-computer interface," Clinical EEG and Neuroscience, vol. 42, no. 4, pp. 253-258, 2011.

41. K. Shindo, K. Kawashima, J. Ushiba, N. Ota, M. Ito, T. Ota, A. Kimura, and M. G. Liu, "Effects of neurofeedback training with an electroencephalogram-based brain-computer 
interface for hand paralysis in patients with chronic stroke: a preliminary case series study," Journal of Rehabilitation Medicine, vol. 43, no. 10, pp. 951-957, 2011.

42. H. Y. Sun, Y. Xiang, and M. D. Yang, "Neurological rehabilitation of stroke patients via motor imaginary-based braincomputer interface technology," Neural Regeneration Research, vol. 6, no. 28, pp. 2198-2202, 2011.

43. K. K. Ang, C. Guan, K. S. Phua, C. Wang, I. Teh, C. W. Chen, and E. Chew, "Transcranial direct current stimulation and EEG-based motor imagery BCI for upper limb stroke rehabilitation," in Proceedings of the 34th Annual International Conference of the IEEE Engineering in Medicine and Biology Society, San Diego, CA, 2012, pp. 4128-4131.

44. E. R. Buch, A. M. Shanechi, A. D. Fourkas, C. Weber, N. Birbaumer, and L. G. Cohen, "Parietofrontal integrity determines neural modulation associated with grasping imagery after stroke," Brain, vol. 135, pp. 596-614, 2012.

45. Y. Kasashima, T. Fujiwara, Y. Matsushika, T. Tsuji, K. Hase, J. Ushiyama, J. Ushiba, and M. G. Liu, "Modulation of event-related desynchronization during motor imagery with transcranial direct current stimulation (tDCS) in patients with chronic hemiparetic stroke," Experimental Brain Research, vol. 221, no. 3, pp. 263-268, 2012.
46. M. Takahashi, K. Takeda, Y. Otaka, R. Osu, T. Hanakawa, M. Gouko, and K. Ito, "Event related desynchronizationmodulated functional electrical stimulation system for stroke rehabilitation: a feasibility study," Journal of Neuroengineering and Rehabilitation, vol. 9, p. 56, 2012.

47. M. Mihara, N. Hattori, M. Hatakenaka, H. Yagura, T. Kawano, T. Hino, and I. Miyai, "Near-infrared spectroscopymediated neurofeedback enhances efficacy of motor imagery-based training in poststroke victims: a pilot study," Stroke, vol. 44, no. 4, pp. 1091-1098, 2013.

48. B. Varkuti, C. Guan, Y. Pan, K. S. Phua, K. K. Ang, C. W. K. Kuah, K. Chua, B. Ti Ang, N. Birbaumer, and R. Sitaram, "Resting state changes in functional connectivity correlate With movement recovery for BCI and robot-assisted upper-extremity training after stroke," Neurorehabilitation and Neural Repair, vol. 27, no. 1, pp. 53-62, 2013.

49. A. Caria, C. Weber, D. Brotz, A. Ramos, L. F. Ticini, A. Gharabaghi, C. Braun, and N. Birbaumer, "Chronic stroke recovery after combined BCI training and physiotherapy: a case report," Psychophysiology, vol. 48, no. 4, pp. 578-582, 2011.

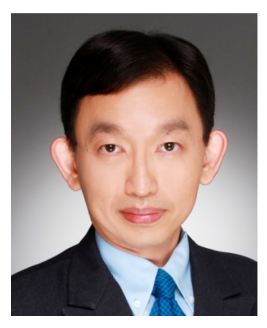

\section{Kai Keng Ang}

Kai Keng Ang received his B.A.Sc. (First Class Hons.) and his Ph.D. degrees in computer engineering from Nanyang Technological University, Singapore. He is currently the Brain-Computer Interface Laboratory Head and a Scientist with the Institute for Infocomm Research, Agency for Science, Technology and Research, Singapore. He was a Senior Software Engineer with Delphi Automotive Systems Singapore Pte. Ltd., from 1999 to 2003, where he was involved in research on embedded software for automotive engine controllers. His current research interests include brain-computer interfaces, computational intelligence, machine learning, pattern recognition, and signal processing.

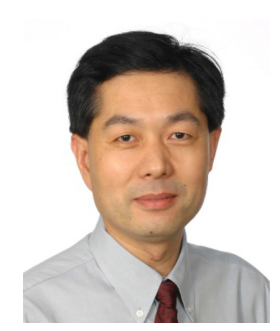

\section{Cuntai Guan}

Dr Cuntai Guan received his Ph.D. degree in Electrical and Electronic Engineering from Southeast University in 1993. He is currently a Principal Scientist and Department Head at the Institute for Infocomm Research, Agency for Science, Technology and Research ( $\left.A^{*} S T A R\right)$, Singapore. His current research interests include neural and biomedical signal processing; neural and cognitive process and its clinical application; braincomputer interface algorithms, systems and its applications. He was the recipient of several awards including g.tec Annual BCI Research Award 2010, IES Prestigious Engineering Achievement Award 2009, etc. He has published over 170 refereed journal and conference papers, and holds 13 granted patents and applications. He delivered over 30 keynote and invited talks. He serves in the editorial board of IEEE Access, IEEE Transactions on Biomedical Engineering, and Frontiers in Neuroprosthetics. He is a senior member of IEEE. 